Ophthalmologe 2022 $\cdot 119: 89-93$

https://doi.org/10.1007/s00347-021-01483-5

Eingegangen: 23. März 2021

Überarbeitet: 15. Juli 2021

Angenommen: 26. Juli 2021

Online publiziert: 27. August 2021

๑ Der/die Autor(en) 2021

\section{Die Kataraktchirurgie und das kleine Auge: Relativer anteriorer Mikrophthalmus, hohe Hyperopie und Nanophthalmus}

Gerd U. Auffarth · Tadas Naujokaitis · Maximilian Hammer

Augenklinik der Universität Heidelberg, Heidelberg, Deutschland

\title{
Zusammenfassung
}

Relativer anteriorer Mikrophthalmus, hochgradige Hyperopie und Nanophthalmus bezeichnen klein gebaute Augen mit unterschiedlichem morphologischem Verhältnis zwischen Vorderabschnitt und Achsenlänge. Im Rahmen dieses Beitrags werden intraoperative Herausforderungen und chirurgische Lösungsansätze für die Kataraktoperation bei Patienten mit einer der 3 genannten morphologischen Veränderungen diskutiert. Zusätzlich wird auf mögliche, vorliegende Komorbiditäten, wie z. B. das Glaukom, und die präoperative Planung eingegangen.

\section{Schlüsselwörter}

Komplizierte Katarakt-Chirurgie · IOL-Berechnung · Einfacher Mikrophthalmos · Phakoemulsifikation - Vorderabschnitt Anatomie · Ko-Morbiditäten

\section{Video Online}

Die Online-Version dieses Beitrags (https:// doi.org/10.1007/s00347-021-01483-5) enthält ein Video.

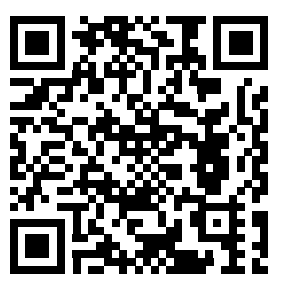

QR-Code scannen \& Beitrag online lesen
Im klinischen Alltag begegnen uns nicht selten „klein gebaute" Augen: Der relative anteriore Mikrophthalmus (RAM), die hochgradige Hyperopie und der Nanophthalmus. Außer der Größenverhältnisse sind die Augen morphologisch intakt und voll funktionsfähig [10]. Diese 3 Krankheitsbilder werden oft unter dem Begriff „einfacher Mikrophthalmus" zusammengefasst und sind abzugrenzen von genetischen Missbildungen mit funktionellen Störungen, die mit einem Mikrophthalmus einhergehen (komplexer Mikrophthalmus). Dieser Beitrag behandelt Techniken und Lösungsansätze für die Kataraktchirurgie bei Patienten mit einfachem Mikrophthalmus. Wird die Morphologie präoperativ erkannt, können Techniken wie die SoftShell-Technik, Posterior-Plane-Emulsifikation, Step-by-Step-Chop, aber auch die angepasste Berechnung der Brechkraft der Intraokularlinse (IOL) zum Einsatz kommen, um bestmögliche Ergebnisse zu erzielen. Die - Tab. 1 gibt eine Übersicht über die Inhalte des Artikels.

\section{Definitionen}

Zur Unterscheidung der Unterformen des einfachen Mikrophthalmus werden 2 Größen herangezogen: die Achsenlänge und die Größe des Vorderabschnitts. Die - Abb. 1 zeigt diese Zusammenhänge auf. Während beim RAM die Achsenlänge nicht auffällig ist ( $>20 \mathrm{~mm}$ ), ist der Vorderabschnitt disproportional klein, definiert durch einen horizontalen Durchmesser der Hornhaut $<11 \mathrm{~mm}$ und einer Tiefe des Vorderabschnitts von rund $2 \mathrm{~mm}$. Liegt ein normaler Vorderabschnitt vor, aber eine verkürzte Achsenlänge, so liegt eine Hyperopie vor. Der Nanophthalmus ist durch ein in der Gesamtheit verkleinertes Auge mit einer Achsenlänge $<20 \mathrm{~mm}$ oder $<20,5 \mathrm{~mm}$ definiert [2].

\section{Relativer anteriorer Mikro- phthalmus}

RAM ist eine häufig nicht erkannte anatomische Besonderheit. Bei ungefähr $6 \%$ der Bevölkerung liegt ein RAM vor, RAM ist damit deutlich häufiger als der Nanophthalmus oder die hochgradige Hype- 
Tab. 1 Übersicht über Herausforderungen der Kataraktchirurgie im „klein gebauten“ Auge

\begin{tabular}{|c|c|}
\hline Herausforderung & Lösungsansatz \\
\hline \multicolumn{2}{|l|}{ IOL-Berechnung } \\
\hline Ungenaue IOL-Berechnung & Patient informieren und Erwartungen anpassen \\
\hline $\begin{array}{l}\text { Hoher postoperativer refraktiver Fehler } \\
\text { wahrscheinlich }\end{array}$ & Korrektion des refraktiven Fehlers postoperativ \\
\hline $\begin{array}{l}\text { Wenige Studien für IOL-Berechnung in } \\
\text { extrem kurzen Augen }\end{array}$ & $\begin{array}{l}\text { Vorsicht bei der Übertragung von Studien in kurzen } \\
\text { Augen auf extrem kurze Augen }\end{array}$ \\
\hline Hohe Diskrepanz zwischen Formeln & Vergleich der Ergebnisse mehrerer Formeln \\
\hline \multicolumn{2}{|l|}{ High-power-Intraokularlinsen } \\
\hline $\begin{array}{l}\text { Toleranz von } \pm 1,0 \mathrm{dpt} \text { für das IOL- } \\
\text { Power-Labeling }\end{array}$ & $\begin{array}{l}\text { Diese Quelle eines postoperativen refraktiven Fehlers } \\
\text { im Kopf behalten }\end{array}$ \\
\hline Erhöhte sphärische Aberration & Verwendung von asphärischen IOLs \\
\hline \multicolumn{2}{|l|}{ Intra- und postoperative Überlegungen } \\
\hline \multirow[t]{2}{*}{$\begin{array}{l}\text { Möglicher lentikulärer Block/ } \\
\text { Glaukomanfall }\end{array}$} & $\begin{array}{l}\text { Präoperative Drucksenkung mit Osmofundin und } \\
\text { Acetazolam (i.v.) }\end{array}$ \\
\hline & Postoperative Kontrolle des intraokularen Drucks \\
\hline Suboptimaler Zugang & Temporaler Zugang \\
\hline Hohe Komplikationsrate & Adäquate Vorbereitung und Informieren des Patienten \\
\hline Erhöhtes Risiko für uveale Effusion & Vollnarkose \\
\hline Hornhautödem & Soft-Shell-Technik \\
\hline Hinterer Kapselriss & Posterior-Plane-Emulsifikation \\
\hline
\end{tabular}

ropie. Bei einem präoperativen Screening müssen Vorderabschnitt und Achsenlänge vermessen werden, um Komplikationen vorzubeugen. Ist ein RAM diagnostiziert, ermöglicht die chirurgische Planung, viele der Probleme zu umgehen und vorbereitet zu sein.

Bei RAM liegt die summierte Prävalenz der Glaukomformen bei $77 \%$. Ein Großteil der Patienten wurde zum Zeitpunkt der Kataraktoperation bereits mit invasiver Glaukomtherapie versorgt. (62\%) Neben den Glaukomen liegen oft Cornea guttata (45\%) und überdurchschnittlich oft hintere Synechien sowie ein Pseudoexfoliationssyndrom vor $(12 \%)[2,10]$.

\section{Hochgradige Hyperopie}

Bei der hochgradigen Hyperopie liegen meist ein morphologisch normaler Vorderabschnitt und ein verkürzter Hinterabschnitt vor. Die Hyperopie kann in 3 Schweregrade, basierend auf dem refraktiven Fehler, eingeteilt werden: geringgradige Hyperopie mit maximal $+2 \mathrm{dpt}$, mittelgradige Hyperopie mit $+2,25 \mathrm{dpt}$ bis $+5 \mathrm{dpt}$ und hochgradige Hyperopie mit über $+5 \mathrm{dpt}$. Die Schwierigkeit der IOL-Berechnung steht somit im Vordergrund. Falls der Hinterabschnitt sehr kurz ist, steigt das Risiko des uvealen Effusi- onssyndroms nach Kataraktchirurgie, eine intraoperative Ablatio ist jedoch sehr selten. Der Übergang zum Nanophthalmus ist fließend.

\section{Nanophthalmus}

Nanophthalmus weist eine sehr geringe Prävalenz mit 0,0009-0,017\% auf. NNO1 auf Chromosom 11 und MFRP auf 11q23.3 sind Gene, die mit Nanophthalmus assoziiert sind. Eine veränderte Kollagenfaseranordnung in diesen Augen führt zu einem erschwerten Wachstum des Auges und einer verdickten und gleichzeitig schwächeren posterioren Sklera, was mit einer Behinderung der venösen Drainage der Vortexvenen mit Risiko einer uvealen Effusion einhergeht. Zusätzlich entsteht durch die normal ausgebildete Linse eine vermehrte Einengung der Vorderkammer, was zum lentikulären Block prädestiniert. Des Weiteren besteht eine erhöhte Wahrscheinlichkeit für intra- und postoperative Komplikationen bei der Kataraktchirurgie in Augen mit kurzer Achsenlänge, wie ein zystisches Makulaödem, choroidale Hämorrhagie, Glaskörperblutungen, Netzhautablösungen, eine Hornhautdekompensation und Kammerwasserabflussstörungen. Unerwünschte Ergebnisse resultieren auf dem
Boden der engen Vorderkammermorphologie, der Komorbiditätslast und durch eine erschwerte Berechnung der Zielrefraktion aufgrund der morphologischen Besonderheiten, auf welche die meisten IOL-Formeln nicht kalibriert sind. Zudem liegt das Auge meist tiefer in der Orbita, was den operativen Zugang erschwert.

Der enge Vorderabschnitt ist dem RAM und dem Nanophthalmus gemein. Im Folgenden werden chirurgische Lösungsansätze hierfür besprochen.

\section{Endothelialer Zellverlust und postoperatives Kornealödem}

Probleme, basierend auf der Enge des Vorderabschnitts, können eingeteilt werden in Komplikationen durch eine enge Morphologie/kleinen Hornhautdurchmesser sowie in Komplikationen durch die kleine Pupillengröße.

In vorherigen Studien trat bei Patienten mit RAM, die bereits Cornea guttata oder eine niedrige Endothelzelldichte zeigten, häufiger ein postoperatives Hornhautödem auf als bei einer Kontrollgruppe mit regelrechtem Vorderabschnitt. Höchstwahrscheinlich wird dies durch die Enge des Vorderabschnitts ausgelöst. Im Durchschnitt verloren die Patienten zwischen 11 und $13 \%$ der Endothelzelldichte. Techniken wie die Soft-Shell-Technik nach Arshinoff [1] ermöglichen einen verbesserten Schutz des Endothels. Dispersive und kohäsive Viskoelastika werden in der Vorderkammer gleichzeitig genutzt: Zuerst wird dispersive, im Anschluss kohäsive Substanz in den Vorderabschnitt gegeben. Es bildet sich ein protektiver Film aus dispersivem Viskoelastikum auf den Endothelzellen aus, während der kohäsive Part die Stabilität (raumfüllender Effekt) in der Vorderkammer gewährleistet. Die Technik bietet generell Vorteile bei engem Vorderabschnitt. Insbesondere die Verwendung von hochkohäsiven OVD (z. B. Healon5, Johnson \& Johnson, New Brunswick, NJ, USA) sind von großer Bedeutung bei einer flachen Vorderkammermorphologie. Durch die hohe Viskosität und Elastizität [3] können eine bessere Stabilität und Tiefe der Vorderkammer, eine Pupillenerweiterung und eine Abflachung der Linsenvorderfläche während der Kataraktoperation erzielt werden. In extremen Fällen mit sehr engem Vor- 


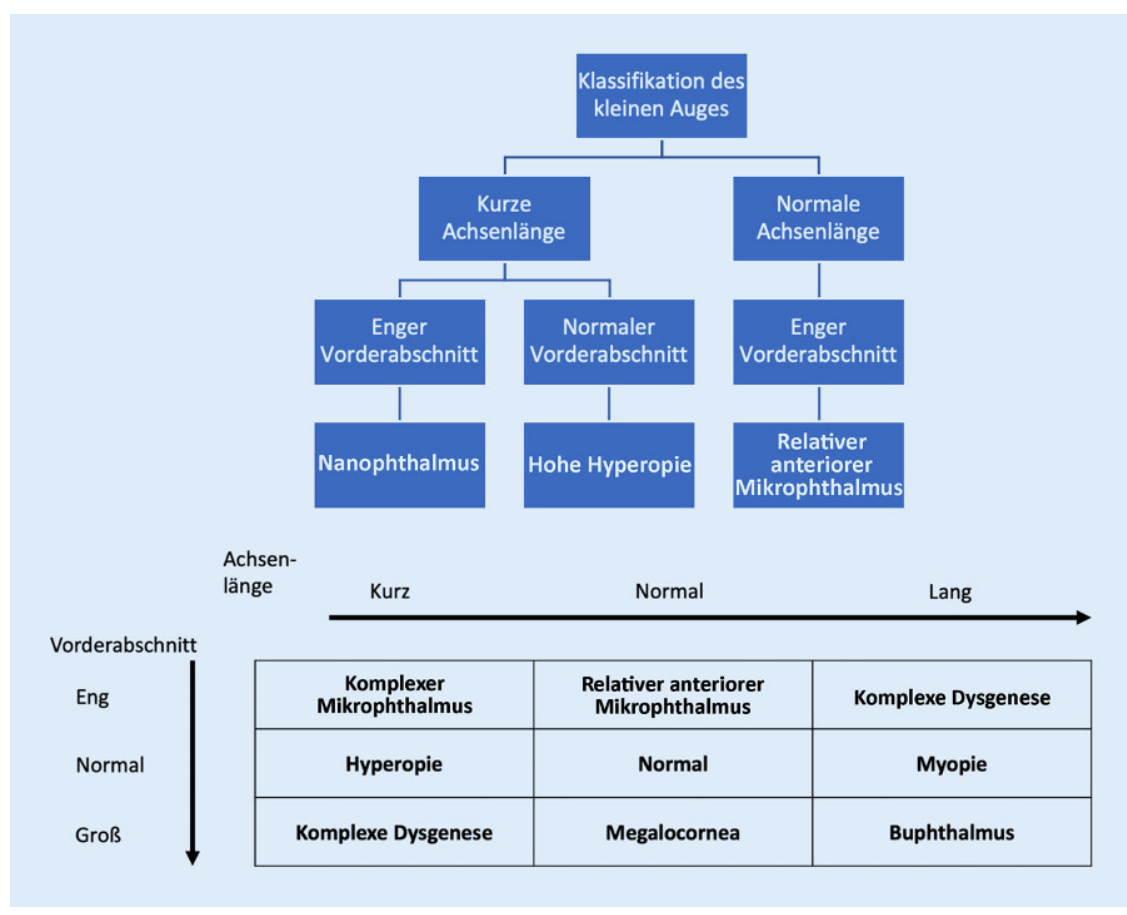

Abb. 1 ム Zusammenhang zwischen Vorderabschnitt und Achsenlänge. (Mod. nach Holladay et al. [6] und Auffarth et al. [2])
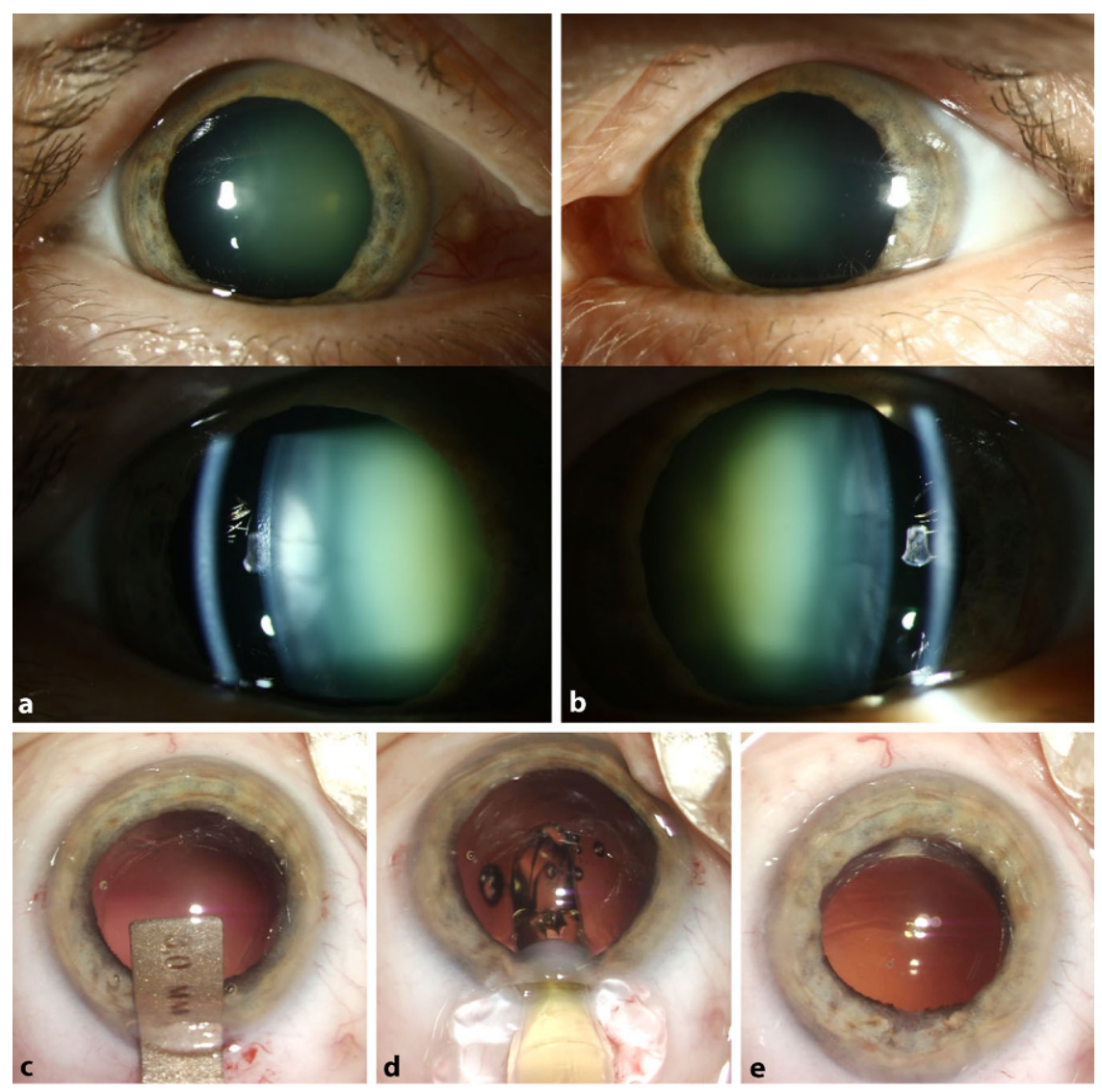

Abb. $2 \Delta$ a, b Präoperative Bilder vom rechten (a) und linken (b) Auge eines Patienten mit Nanophthalmus.c, d, e Eine 3-mm-Inzision temporal wurde gewählt, um die hydrophobe 56-dpt-Linse problemlos implantieren zu können. (Abbildung mod. nach Naujokaitis et al. [9]) derabschnitt empfiehlt es sich, eine vordere Vitrektomie zu Beginn der Operation durchzuführen. Die dadurch verbesserte Situation ermöglicht einen komplikationsärmeren Verlauf.

Durch den engen Vorderabschnitt besteht ebenso ein erhöhtes Risiko für einen Einriss der hinteren Kapsel: Meist versucht der Chirurg instinktiv, das Endothel zu schützen, und aspiriert Teile der Linse weiter posterior als gewöhnlich. Vasavada et al. liefern eine mögliche Lösung: die Posterior-Plane-Emulsifikation mit Sculpting [13]. Während des Vorgangs werden gleichzeitig Stärke des Vakuums, Energie und Fluss der Aspiration verringert, um effektiv Schäden der hinteren Kapsel zu vermeiden. Eine Hinterkapselruptur ist in dieser Gruppe an Patienten strengstens zu vermeiden. Die meist individuell hergestellten IOLs liegen in der Regel als einstückige IOLs zur geplanten Kapselsackimplantation vor. Sollte eine Sulkusimplantation nötig werden, kann diese nicht mit der einstückigen IOL durchgeführt werden. Bis zur endgültigen Fixation einer neu gefertigten High-Power-IOL finden Standby-Strategien Anwendung.

\section{Enger Pupillendurchmesser und Pseudoexfoliation}

Die hohe Prävalenz einer kleinen Pupille $(<4 \mathrm{~mm})$ in Kombination mit hinteren Synechien und Pseudoexfoliationssyndrom in dieser Patientenpopulation macht eine mechanische Dilatation der Pupille oft unabwendbar.

Reicht die pharmakologische Dilatation der Pupille während der Operation nicht aus, gibt es mehrere Möglichkeiten zur mechanischen Dilatation: Sphinkterotomie, Irisretraktoren und Pupillenexpander (i-Ring, Malyugin-Ring). Studien zeigten, dass bei richtiger Verwendung eines Irisexpanders hierbei sogar Komplikationen wie Endothelzellverlust verringert werden können.

Trotz mechanischer Dilatation werden in diesen Augen selten optimale Bedingungen geschaffen. Bei der Phakoemulsifikation kann z. B. die Step-by-Step-ChopTechnik nach Vasavada verwendet werden. Die geringstmögliche Manipulation der Iris ist wichtig, da schon vor der Kataraktoperation besonders bei Patienten 
Tab. 2 Vergleich verschiedener IOL-Berechnung im Patientenbeispiel mit Nanophthalmus. (Aus Naujokaitis et al. [9])

\begin{tabular}{|l|l|l|}
\hline Formel & Rechtes Auge & Linkes Auge \\
\hline
\end{tabular}

Berechnete IOL-Power für Emmetropia

\begin{tabular}{|l|l|l|} 
Hoffer Q & $+70,09 \mathrm{dpt}$ & $+69,96 \mathrm{dpt}$ \\
\hline Haigis & $+55,28 \mathrm{dpt}$ & $+57,47 \mathrm{dpt}$ \\
\hline SRK/T & $+56,04 \mathrm{dpt}$ & $+57,09 \mathrm{dpt}$ \\
\hline Holladay 1 & $+57,07 \mathrm{dpt}$ & $+59,20 \mathrm{dpt}$ \\
\hline Holladay 2 & $+57,43 \mathrm{dpt}$ & $+59,05 \mathrm{dpt}$
\end{tabular}

Fehler der Vorhersage (postoperatives sphärisches Äquivalent - Zielrefraktion)

\begin{tabular}{|l|l|l|}
\hline Hoffer Q & $-7,57 \mathrm{dpt}$ & $-7,75 \mathrm{dpt}$ \\
\hline Haigis & $+1,21 \mathrm{dpt}$ & $+0,06 \mathrm{dpt}$ \\
\hline SRK/T & $+0,60 \mathrm{dpt}$ & $+0,34 \mathrm{dpt}$ \\
\hline Holladay 1 & $-0,19 \mathrm{dpt}$ & $-1,29 \mathrm{dpt}$ \\
\hline Holladay 2 & $-0,45 \mathrm{dpt}$ & $-1,18 \mathrm{dpt}$ \\
\hline
\end{tabular}

Große Abweichungen zwischen den Formeln sind ersichtlich

Der Vergleich der Formeln erlaubt eine bessere Einschätzung der nötigen Brechkraft der Kunstlinse

mit RAM eine ausgeprägte Irisinstabilität vorliegen kann.

\section{Komorbiditätslast: Implikationen für die Kataraktchirurgie}

\section{Glaukome}

Vom Engwinkel- bis zum Offenwinkelglaukom (z. B. sekundäres Pseudoexfoliationsglaukom) können verschiedene Formen des Glaukoms vorliegen. In vielen Fällen mit engem Vorderabschnitt wurde bereits ein Glaukom operativ therapiert. In diesen Fällen ist ein temporaler Zugangsweg oft vorteilhaft. Studien zeigten, dass hierdurch möglicherweise auch ein geringerer Verlust an Hornhautendothel möglich ist. In bestimmten Formen des Nanophthalmus kann ein Glaukomanfall als lentikulärer Block durch die natürliche Linse selbst (und später durch die Kunstlinse) ausgelöst werden. Die Kataraktoperation kann hier als "kausale" Therapie des Glaukoms erwogen werden, bevor invasive Glaukomverfahren Anwendung finden (antiglaukomatöse Linsenextraktion).

\section{IOL-Berechnung}

Das gute postoperative, refraktive Ergebnis ist in kurz gebauten Augen eine Her- ausforderung. Seit 1993 wird die HofferQ-Formel als Ausgangspunkt für die IOLBerechnung in kurzen Augen angesehen [11]. Eine Studie von 2016 in 29 Augen mit RAM zeigte eine postoperative Zielrefraktion \pm 1 dpt in $72,4 \%$ mit der HofferQ-Formel. Dies war nur in 46,6\% der Fälle bei den 15 eingeschlossenen, nanophthalmischen Augen der Fall [7].

Eine Reihe an aktuellen Studien konnte keine signifikanten Unterschiede zwischen Hoffer Q, Barrett Universal II, Haigis, Holladay 2 und RBF 1.0 bei der IOL-Berechnung in kurzen Augen zeigen [12]. Es wird empfohlen, bei Patienten mit flacher Vorderkammertiefe (z. B. $<2,4 \mathrm{~mm}$ ) dies zu beachten. Je flacher die Vorderkammer, desto ungenauer war die IOL-Berechnung basierend auf Hoffer-Q- und Haigis-Formel [4].

Eine neuere Formel, die ebenfalls vielversprechende Ergebnisse in kurzen Augen, insbesondere bei hochgradiger Hyperopie, erzielen konnte, ist die Kane-Formel [8]. Hipólito-Fernandes et al. zeigten, dass unter anderem die Kane-Formel in geringem Maße in ihrem postoperativen Ergebnis durch die Linsendicke und Vorderkammertiefe beeinflusst wird. Im Gegensatz dazu neigte die z. B. Haigis-Formel zu refraktiven Abweichungen bei variierenden Linsendicken [5]. Die Kane-Formel sollte jedoch nur in Kombination mit etablierten Formeln zum Einsatz kommen. Sie wurde erst im September 2017 entwickelt und ist nicht offengelegt, was die externe Validierung erschwert.

Insbesondere bei Patienten mit Nanophthalmus sollten die Ergebnisse mehrerer Formeln verglichen werden. Hierbei können Ausreißer identifiziert werden, die bei alleiniger Verwendung einer Formel zu großen refraktiven Fehlern führen können. Für RAM, Nanophthalmus und hochgradige Hyperopie empfiehlt sich die Verwendung von High-Power-IOLs, die zum Teil individuell hergestellt werden. Die Verwendung von Piggyback-IOL-Implantaten ist in Deutschland obsolet und sollte nur in Regionen angewendet werden, in denen High-Power-IOLs nicht verfügbar sind. Eine der möglichen Fehlerquellen der postoperativen Refraktion basiert jedoch auch auf den High-Power-IOLs: Die zugehörige ISO-Norm erlaubt eine höhere Toleranz der Brechkraft bei Linsen mit hohen Brechwer- ten und sollte als potenzieller Fehler beachtet werden.

Video 1 zeigt ein Beispiel für eine gelungene Kataraktoperation bei einem 60-jährigen Patienten mit bilateralem Nanophthalmus (- Abb. 2). Als zusätzliche Technik wurde ein Femtosekundenlaser für die Kapsulotomie und Fragmentierung der Linse verwendet. Die Hauptinzision wurde temporal platziert. Vor der Operation wurde mithilfe des IOLMaster 700 (Carl Zeiss AG, Oberkochen, Deutschland) die benötigte Brechung der Linse nach mehreren Formeln berechnet (Haigis, Holladay 1, Holladay 2, SRK/T). Die Ergebnisse der Formeln zeigten gravierende Unterschiede, was die Wichtigkeit des Vergleichs mehrerer Formeln bei kleinen Augen aufzeigt (•Tab. 2). Eine Linse mit +56 dpt wurde gewählt. Der postoperative Verlauf gestaltete sich problemlos. Dieser kurze Fallbericht soll zeigen, dass die Kataraktchirurgie bei nanophthalmischen Augen bei gründlicher Vorbereitung komplikationsfrei und erfolgreich durchgeführt werden kann. Einen ausführlichen Fallbericht der Operationen präsentiert Naujokaitis et al. [9].

\section{Fazit für die Praxis}

- Eine Messung der Achsenlänge sowie der Vorderkammertiefe ist präoperativ nötig, um Patienten mit veränderter Vorderkammermorphologie zu erkennen (Scheimpflug, Vorderabschnitts-OCT).

- Step-by-Step-Chop, Posterior-Plane-Emulsifikation und Soft-Shell-Technik sind Methoden, um die Phakoemulsifikation sicherer zu gestalten und den Endothelzellverlust zu senken.

- Modifizierte Formeln für kurze Achsenlängen sollten zur IOL-Planung verwendet werden und miteinander verglichen werden.

- Die Kataraktoperation in klein gebauten Augen sollte von erfahrenen Chirurgen durchgeführt werden. 


\section{Korrespondenzadresse}

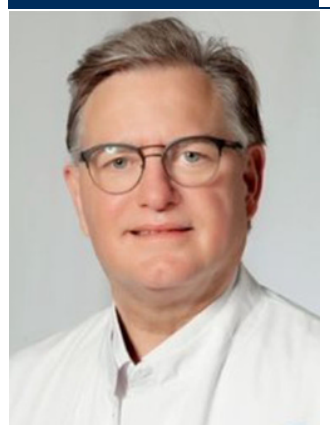

\section{Gerd U. Auffarth}

Augenklinik der Universität Heidelberg Im Neuenheimer Feld 400, 69120 Heidelberg, Deutschland

Gerd.auffarth@med.uni-heidelberg.de

\section{Einhaltung ethischer Richtlinien}

Interessenkonflikt. G.U. Auffarth, T. Naujokaitis und M. Hammer geben an, dass kein Interessenkonflikt besteht.

Dieser Artikel enthält keine Informationen, die zur Identifikation eines Patienten führen können. Die retrospektive Bearbeitung des Falles wurde gemäß der Deklaration von Helsinki durchgeführt.

Open Access. Dieser Artikel wird unter der Creative Commons Namensnennung 4.0 International Lizenz veröffentlicht, welche die Nutzung, Vervielfältigung, Bearbeitung, Verbreitung und Wiedergabe in jeglichem Medium und Format erlaubt, sofern Sie den/die ursprünglichen Autor(en) und die Quelle ordnungsgemäß nennen, einen Link zur Creative Commons Lizenz beifügen und angeben, ob Änderungen vorgenommen wurden.

Die in diesem Artikel enthaltenen Bilder und sonstiges Drittmaterial unterliegen ebenfalls der genannten Creative Commons Lizenz, sofern sich aus der Abbildungslegende nichts anderes ergibt. Sofern das betreffende Material nicht unter der genannten Creative Commons Lizenz steht und die betreffende Handlung nicht nach gesetzlichen Vorschriften erlaubt ist, ist für die oben aufgeführten Weiterverwendungen des Materials die Einwilligung des jeweiligen Rechteinhabers einzuholen.

Weitere Details zur Lizenz entnehmen Sie bitte der Lizenzinformation auf http://creativecommons.org/ licenses/by/4.0/deed.de.

\section{Literatur}

1. Arshinoff SA (1999) Dispersive-cohesive viscoelastic soft shell technique. J Cataract Refract Surg 25:167-173. https://doi.org/10.1016/s08863350(99)80121-7

2. Auffarth GU, Blum M, Faller U et al (2000) Relative anterior microphthalmos: morphometric analysis and its implications for cataract surgery. Ophthalmology 107:1555-1560. https://doi.org/ 10.1016/s0161-6420(00)00240-2

\section{Cataract surgery and the small eye: relative anterior microphthalmos, high hyperopia and nanophthalmos}

Relative anterior microphthalmos, nanophthalmos and high-grade hyperopia are small eyes with different characteristic morphological relationships between the anterior segment and axis length. This article discusses the intraoperative challenges and surgical approaches to solutions for cataract operations in patients with one of the three named morphological alterations. Additionally, the article addresses possible comorbidities including glaucoma and preoperative planning.

\section{Keywords}

Complex cataract surgery $\cdot$ IOL calculation - Simple microphthalmos $\cdot$ Phacoemulsification . Anterior segment anatomy · Comorbidities

3. Dick HB, Krummenauer F, Augustin AJ et al (2001) Healon 5 viscoadaptive formulation: comparison to Healon and Healon GV. J Cataract Refract Surg 27:320-326. https://doi.org/10.1016/s08863350(00)00482-X

4. Eom Y, Kang S-Y, Song JS et al (2014) Comparison of Hoffer $Q$ and Haigis formulae for intraocular lens power calculation according to the anterior chamber depth in short eyes. Am J Ophthalmol 157:818-824.e2. https://doi.org/10.1016/j.ajo. 2013.12.017

5. Hipólito-Fernandes D, Luís ME, Serras-Pereira Retal (2020) Anterior chamber depth, lens thickness and intraocular lens calculation formula accuracy: nine formulas comparison. BrJOphthalmol. https://doi. org/10.1136/bjophthalmol-2020-317822

6. Holladay JT, Gills JP, Leidlein J, Cherchio M (1996) Achieving emmetropia in extremely short eyes with two piggyback posterior chamber intraocular lenses. Ophthalmology 103:1118-1123. https:// doi.org/10.1016/s0161-6420(96)30558-7

7. Jung KI, Yang JW, Lee YC, Kim S-Y (2012) Cataract surgery in eyes with nanophthalmos and relative anterior microphthalmos. Am J Ophthalmol 153:1161-1168.e1. https://doi.org/10.1016/j.ajo. 2011.12.006

8. Kane JX, Melles RB (2020) Intraocular lens formula comparison in axial hyperopia with a high-power intraocular lens of 30 or more diopters. J Cataract Refract Surg 46:1236-1239. https://doi.org/10. 1097/j.jcrs.0000000000000235

9. Naujokaitis T, Scharf D, Baur l et al (2020) Bilateral implantation of +56 and +58 diopter custommade intraocular lenses in patient with extreme nanophthalmos. Am J Ophthalmol Case Rep 20:100963. https://doi.org/10.1016/j.ajoc.2020. 100963

10. Nihalani BR, Jani UD, Vasavada AR, Auffarth GU (2005) Cataract surgery in relative anterior microphthalmos. Ophthalmology 112:1360-1367. https://doi.org/10.1016/j.ophtha.2005.02.027

11. Savini G, Taroni L, Hoffer KJ (2020) Recent developments in intraocular lens power calculation methods - update 2020. Ann Transl Med 8:1553. https://doi.org/10.21037/atm-20-2290

12. Shrivastava AK, Behera P, Kumar B, Nanda S (2018) Precision of intraocular lens power prediction in eyes shorter than $22 \mathrm{~mm}$ : an analysis of 6 formulas. J Cataract Refract Surg 44:1317-1320. https://doi. org/10.1016/j.jcrs.2018.07.023

13. Vasavada AR, Raj S (2003) Step-down technique. J Cataract Refract Surg 29:1077-1079. https://doi. org/10.1016/s0886-3350(03)00121-4 